\title{
X Congreso Ibérico de Agroingeniería

\section{Evaluación de los efectos de la aplicación de biochar como enmienda orgánica en un cultivo de sorgo (Sorghum bicolor L.) bajo condiciones de invernadero}

\author{
M. Videgain ${ }^{1}$, P. Marco ${ }^{2}$, C. Martí1 ${ }^{1}$ F.J. García-Ramos' ${ }^{1}$ J.J. Manyà ${ }^{1}$, M.C. Jaizme-Vega ${ }^{3}$ \\ 1 Escuela Politécnica Superior de Huesca. Universidad de Zaragoza; mvidegain@unizar.es \\ 2 Centro de Investigación y Tecnología Agroalimentaria de Aragón. \\ 3 Instituto Canario de Investigaciones Agrarias
}

Resumen: La aplicación como enmienda orgánica en el suelo de biochar procedente de sarmiento de vid (Vitis vinifera L.) supone una alternativa de gestión agrícola de interés. El comportamiento de este tipo de biochar en el suelo precisa de una mayor información que permita ajustar las condiciones de pirólisis con el objetivo de obtener un biochar de calidad. En este trabajo se ha desarrollado un ensayo agronómico con la finalidad de evaluar la influencia de la aplicación de este tipo de biochar sobre el sistema suelo-planta-microorganismos. Se ha establecido un diseño tri-factorial en bloques al azar teniendo en cuenta como factores la temperatura final de pirólisis $\left(400^{\circ} \mathrm{C}\right.$ y $\left.600{ }^{\circ} \mathrm{C}\right)$, la dosis de aplicación $(20 \mathrm{t} / \mathrm{ha}$ y $40 \mathrm{t} / \mathrm{ha}$ ) y la textura del sustrato (franco-arenosa y franco-arcillosa). El cultivo seleccionado ha sido sorgo (Sorghum bicolor L.) cuyo desarrollo y producción se han evaluado durante dos ciclos completos de cultivo. Como indicador biológico se han cuantificado algunas poblaciones microbianas. Se han observado diferencias significativas en el sustrato franco-arenoso: la aplicación de biochar producido a $400{ }^{\circ} \mathrm{C}$ ha incrementado significativamente la biomasa seca de las raíces de las plantas en la segunda cosecha (47,5\% respecto al control). Además, en este mismo sustrato, la aplicación del biochar producido a $600{ }^{\circ} \mathrm{C}$, independientemente de la dosis, ha incrementado el número de poblaciones de hongos filamentosos respecto al tratamiento control. Los microorganismos aeróbios mesófilos totales se incrementaron en ambos tipos de sustrato con la adición de biochar.

Palabras clave: pirólisis, sarmiento de vid, poblaciones microbianas.

\section{Introducción}

Existen diversas fuentes de materia orgánica para utilizar como enmiendas en suelos, entre ellas aparece el biochar. El término biochar se define como un material carbonoso obtenido a partir de biomasa mediante descomposición térmica a temperaturas relativamente bajas $\left(<700{ }^{\circ} \mathrm{C}\right)$ y baja o nula concentración de oxígeno, en un proceso conocido como pirólisis [1].

Algunos organismos internacionales que trabajan en la promoción de este material pirogénico, y científicos que trabajan en su estudio [2] proponen en su definición la particularidad de denominar al material pirolizado como biochar cuando su destino es la aplicación como enmienda orgánica de suelos y para el secuestro de carbono en los mismos, siendo estos últimos aspectos de gran importancia, ya que se descarta su uso como combustible. 


\section{CONGRESO IBÉRICO DE AGROINGENIERÍA \\ X CONGRESSO IBÉRICO DE AGROENGENHARIA \\ 3 - 6 septiembre 2019, Huesca - España}

El uso de biochar supone una alternativa de gestión ambiental que está adquiriendo gran repercusión en los últimos años, ya que puede ser una vía de actuación simultánea en varios frentes: mejorar la productividad de suelos agrícolas, valorizar residuos y actuar como sumidero de $\mathrm{CO}_{2}$. El biochar puede actuar como mejorante de suelos aumentando su fertilidad como consecuencia del aumento de la capacidad de intercambio catiónico de los mismos y del aumento del $\mathrm{pH}$ [3] en suelos ácidos. La utilización de biochar lleva asociados beneficios ambientales potenciales, gracias al aumento de la capacidad de retención de agua y de nutrientes y la consecuente mejora de la calidad del agua (reducción de lixiviados). El carbón vegetal obtenido mediante pirólisis es una sustancia estable rica en carbono, cuya aplicación en suelos comporta una retención o secuestro de carbono. Algunos autores se refieren al uso del biochar como una estrategia que implica un ciclo negativo de carbono y, por lo tanto, la vía más interesante para reducir las emisiones de $\mathrm{CO}_{2}$ mediante el uso de biomasa $[4,5]$.

El grado de conocimiento sobre el efecto del biochar en el sistema suelo-plantamicroorganismos es muy reciente y los resultados consultados son muy variables y dependientes del tipo de experimento llevado a cabo.

Las propiedades finales del biochar dependen de las condiciones de trabajo del proceso de pirólisis (temperatura final de producción, presión y tiempo de residencia del gas en el interior del reactor) que su vez también tienen una influencia clara sobre el rendimiento del proceso [6-9]. Además de estas condiciones es necesario tener en cuenta el tipo de biomasa utilizada (composición, humedad y granulometría) [10,11], que además de influir sobre el propio proceso de pirólisis, tendrá un comportamiento muy diferente en el suelo como enmienda orgánica en función de sus características intrínsecas.

Con el objetivo de producir un biochar de calidad a partir de la revalorización de subproductos agrarios, en el Grupo de Investigación en Biochar de la Escuela Politécnica de Huesca se ha trabajado de forma experimental en la producción de biochar a partir de varios tipos de biomasa (restos de poda de viñedo, alperujo de oliva, cañote de maíz, paja de trigo, restos de poda de encina, entre otros). Debido al volumen de residuos que ofrece la poda del viñedo [12], así como la problemática de la aplicación directa al suelo en función de las condiciones de cada explotación [13], se ha considerado interesante profundizar en las posibilidades de su proceso de transformación en biochar, teniendo en cuenta la información existente sobre las condiciones de operación para este proceso pero atendiendo a la falta de información sobre sus efectos al ser aplicado al suelo.

El objetivo de este trabajo es evaluar los efectos de la aplicación de biochar de sarmiento de vid sobre el desarrollo de un cultivo de sorgo bajo condiciones controladas de invernadero tomando como indicador biológico el comportamiento de algunas comunidades microbianas del suelo.

\section{Materiales y métodos}

\subsection{Recogida y caracterización de biomasa}

El sarmiento utilizado para la producción de biochar procede de la poda invernal de plantas de vid (Vitis vinifera) y fue recogido en una parcela de una bodega de vino situada en la comarca del Somontano de Huesca, en el noreste de Aragón. La variedad de vid fue Cabernet Sauvignon en una etapa de máxima producción y un índice de Ravaz 6.

Una vez recolectada, la biomasa se almacenó en cajas de cartón para su transporte a laboratorio. Los sarmientos fueron seleccionados por su diámetro (entre 8,5 y $15,0 \mathrm{~mm}$ ) y se cortaron con tijera de poda en piezas de aproximadamente $40 \mathrm{~mm}$ de longitud con la intención de maximizar la eficiencia del proceso de pirólisis (tanto el contenido de carbono fijo en el biochar como el rendimiento del proceso), relacionada con el tamaño de partícula inicial $[14,15]$. 


\section{CONGRESO IBÉRICO DE AGROINGENIERÍA \\ X CONGRESSO IBÉRICO DE AGROENGENHARIA \\ 3 - 6 septiembre 2019, Huesca - España}

Tras un muestreo representativo de la biomasa se realizó el análisis inmediato siguiendo el procedimiento estandarizado ASTM (D3173 para la humedad, D3174 para las cenizas y D3175 para la materia volátil). El análisis elemental de la biomasa se realizó mediante un analizador elemental LECO micro CHNS en el laboratorio del Grupo de Procesos Termoquímicos de la Universidad de Zaragoza. Por último, la composición de la ceniza se analizó de acuerdo a la norma ASTM D4326-04 a través de fluorescencia de rayos X (XRF). La Tabla 1 refleja los resultados de la caracterización de la biomasa inicial.

Tabla 1. Análisis inmediato, elemental y XRF del sarmiento recolectado

\begin{tabular}{|c|c|c|c|}
\hline \multicolumn{2}{|c|}{ Inmediato (\% p/p) } & \multicolumn{2}{|c|}{$\begin{array}{c}\text { Elemental ( } \% \mathrm{p} / \mathrm{p} \text { en base seca } \\
\text { y libre de cenizas) }\end{array}$} \\
\hline Ceniza & $2,46 \pm 0,37$ & $\mathrm{C}$ & $42,29 \pm 0,49$ \\
\hline Humedad & $10,47 \pm 0,13$ & $\mathrm{H}$ & $5,24 \pm 0,06$ \\
\hline Volátiles & $72,93 \pm 1,64$ & $\mathrm{~N}$ & $13,52 \pm 0,35$ \\
\hline Carbono fijo & $14,14 \pm 1,38$ & $\mathrm{O}$ & $38,96 \pm 0,45$ \\
\hline \multicolumn{4}{|c|}{ Compuestos inorgánicos ( $\%$ p/p sobre cenizas) } \\
\hline $\mathrm{CaO}$ & 58,30 & $\mathrm{PbO}$ & 0,26 \\
\hline $\mathrm{K} 2 \mathrm{O}$ & 18,40 & $\mathrm{SnO} 2$ & 0,26 \\
\hline $\mathrm{SiO} 2$ & 5,73 & $\mathrm{CuO}$ & 0,09 \\
\hline $\mathrm{Fe} 2 \mathrm{O} 3$ & 3,51 & $\mathrm{MnO}$ & 0,53 \\
\hline $\mathrm{Al} 2 \mathrm{O} 3$ & 2,57 & $\mathrm{ZnO}$ & 0,33 \\
\hline $\mathrm{P} 2 \mathrm{O} 5$ & 1,24 & $\mathrm{SO} 3$ & 0,60 \\
\hline $\mathrm{MgO}$ & 6,66 & $\mathrm{Cl}$ & 0,47 \\
\hline $\mathrm{TiO} 2$ & 0,34 & & \\
\hline
\end{tabular}

\subsection{Producción y caracterización de biochar}

Los experimentos de pirólisis se llevaron a cabo en un reactor de lecho fijo a escala de laboratorio situado en la Escuela Politécnica Superior de Huesca. Los detalles sobre la configuración específica del reactor, el proceso de pirólisis y la metodología de análisis de los gases producto de la pirólisis pueden consultarse en publicaciones previas [10,16]. Se realizaron un total de 13 experimentos a dos temperaturas diferentes $\left(400-600{ }^{\circ} \mathrm{C}\right)$ a presión atmosférica, con un caudal constante de 0,6 $1 \mathrm{~min}^{-1}$ de $\mathrm{N}_{2}$ y un mismo tiempo de residencia del gas en el interior del reactor (180 s). En la Tabla 2 se detalla la producción de biochar total y el rendimiento medio de biomasa a biochar de los experimentos.

Tabla 2. Rendimiento medio del proceso de pirólisis en la producción de biochar a diferentes temperaturas $\left(400^{\circ} \mathrm{C}-\mathrm{B} 400\right.$ y $\left.600^{\circ} \mathrm{C}-\mathrm{B} 600\right)$

\begin{tabular}{cccccc}
\hline $\begin{array}{c}\text { Tipo de } \\
\text { biochar }\end{array}$ & $\begin{array}{c}\text { No } \\
\text { experimentos } \\
\text { realizados }\end{array}$ & $\begin{array}{c}\text { Temperatura } \\
\text { de pirólisis } \\
\left({ }^{\circ} \mathbf{C}\right)\end{array}$ & $\begin{array}{c}\text { Total } \\
\text { biomasa } \\
\text { utilizada }(\mathrm{g})\end{array}$ & $\begin{array}{c}\text { Total biochar } \\
\text { producido }(\mathrm{g})\end{array}$ & $\begin{array}{c}\text { Rendimiento } \\
\text { medio biochar }\end{array}$ \\
\hline B400 & 8 & 400 & $2.536,52$ & 959,63 & 0,38 \\
B600 & 5 & 600 & $4.083,69$ & $1.206,74$ & 0,29 \\
\hline
\end{tabular}

Se realizaron análisis inmediato y elemental sobre los diferentes tipos de biochar obtenido siguiendo las normas especificadas en el apartado anterior. Además, se enviaron muestras del producto final al Laboratorio Agroambiental del Gobierno de Aragón donde se realizaron los análisis de referencia para enmiendas orgánicas. Los resultados obtenidos se detallan en la Tabla 3. 
X CONGRESO IBÉRICO DE AGROINGENIERÍA

X CONGRESSO IBÉRICO DE AGROENGENHARIA

3 - 6 septiembre 2019, Huesca - España

Tabla 3. Análisis inmediato, elemental y nutrientes en los dos tipos de biochar $\left(400^{\circ} \mathrm{C}-\mathrm{B} 400 \mathrm{y}\right.$ $\left.600{ }^{\circ} \mathrm{C}-\mathrm{B} 600\right)$

\begin{tabular}{cccccc}
\hline & Inmediato (\% p/p) & \multicolumn{3}{c}{ Elemental (\% p/p en base seca y libre de cenizas) } \\
\hline & $\mathbf{B 4 0 0}$ & $\mathbf{B 6 0 0}$ & & $\mathbf{B} 400$ & $\mathbf{B 6 0 0}$ \\
\hline Ceniza & $6,45 \pm 0,14$ & $10,02 \pm 0,81$ & $\mathrm{C}$ & $71,54 \pm 0,48$ & $82,89 \pm 0,33$ \\
Humedad & $0,57 \pm 0,22$ & $3,05 \pm 0,34$ & $\mathrm{H}$ & $4,46 \pm 0,19$ & $1,95 \pm 0,08$ \\
Volátiles & $22,03 \pm 0,01$ & $2,39 \pm 0,71$ & $\mathrm{~N}$ & $1,58 \pm 0,10$ & $1,52 \pm 0,01$ \\
Carbono fijo & $70,88 \pm 6,45$ & $84,54 \pm 1,32$ & $\mathrm{O}$ & $22,42 \pm 0,77$ & $13,63 \pm 0,31$ \\
\hline
\end{tabular}

Nutrientes (análisis de referencia para abono orgánico)

\begin{tabular}{|c|c|c|c|c|c|c|c|c|c|}
\hline \multirow{2}{*}{$\begin{array}{l}\text { Nutrientes } \\
\text { principales }\end{array}$} & \multirow{2}{*}{ Método } & \multirow{2}{*}{ Unidad } & \multicolumn{2}{|c|}{ Resultado } & \multirow{2}{*}{$\begin{array}{l}\text { Nutrientes } \\
\text { secundarios }\end{array}$} & \multirow{2}{*}{ Método } & \multirow{2}{*}{ Unidad } & \multicolumn{2}{|c|}{ Resultado } \\
\hline & & & B400 & B600 & & & & B400 & B600 \\
\hline $\begin{array}{l}\text { Nitrógeno total } \\
\text { Kjeldahl }\end{array}$ & $\begin{array}{l}\text { MT-FER- } \\
001\end{array}$ & $\mathrm{p} / \mathrm{n}$ & 1,40 & 1,20 & $\begin{array}{l}\text { Calcio total } \\
(\mathrm{CaO})\end{array}$ & P-OES & $\mathrm{p}$ & 2,90 & 3,80 \\
\hline $\begin{array}{l}\text { Fósforo total } \\
\qquad\left(\mathrm{P}_{2} \mathrm{O}_{5}\right)\end{array}$ & $\begin{array}{l}\text { ULTRAV. } \\
\text { VISIBLE }\end{array}$ & $\mathrm{p} / \mathrm{p}$ & 2,08 & 2,45 & $\begin{array}{l}\text { Magnesio total } \\
\qquad(\mathrm{MgO})\end{array}$ & ICP-OES & $\% \mathrm{p} / \mathrm{p}$ & 0,68 & 0,79 \\
\hline $\begin{array}{l}\text { Potasio total } \\
\qquad\left(\mathrm{K}_{2} \mathrm{O}\right)\end{array}$ & ICP-OES & & 1 & 2,10 & $\begin{array}{l}\text { Sodio total } \\
\left(\mathrm{Na}_{2} \mathrm{O}\right)\end{array}$ & & & 50,00 & 540,00 \\
\hline \multirow[b]{2}{*}{ Microelementos } & \multirow[b]{2}{*}{ Método } & \multirow[b]{2}{*}{ Unidad } & \multicolumn{2}{|c|}{ Resultado } & \multirow{2}{*}{$\begin{array}{c}\text { Caract. } \\
\text { Físico- } \\
\text { químicas }\end{array}$} & \multirow[b]{2}{*}{ Método } & \multirow[b]{2}{*}{ Unidad } & \multicolumn{2}{|c|}{ Resultado } \\
\hline & & & B400 & B600 & & & & B400 & B600 \\
\hline Hierr & ICP-OES & 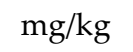 & 280 & 210 & Mat. orgánica & Calcinación & $\% \mathrm{p} / \mathrm{p}$ & 86,40 & 85,40 \\
\hline Col & IO & $\mathrm{mg} / \mathrm{kg}$ & 43 & 38 & $\begin{array}{l}\text { Densidad } \\
\text { aparente }\end{array}$ & & $n^{3}$ & 0,33 & 0,40 \\
\hline Mangan & ICP-OES & & 100 & 102 & & & & & \\
\hline Cinc to & ICP-OES & $\mathrm{mg} / \mathrm{kg}$ & 145 & 135 & & & & & \\
\hline
\end{tabular}

El biochar obtenido se procesó mecánicamente a través de un removedor manual diseñado específicamente para simular las condiciones de movimiento del producto en el sistema de distribución de una abonadora convencional. Como resultado de este proceso se obtuvieron diferentes fracciones de tamaños que se guardaron por separado para respetar, de una forma aproximada, los porcentajes obtenidos a la hora de aplicar el biochar en las macetas del ensayo.

La distribución media del tamaño del producto preparado para su aplicación en el ensayo se detalla en la Tabla 4:

Tabla 4. Distribución media del tamaño de partícula tras el procesado mecánico del biochar con removedor manual para las dos temperaturas de pirólisis

\begin{tabular}{ccc}
\hline Fracción & B400 (\%) & B600 (\%) \\
\hline$<2 \mathrm{~mm}$ & 7 & 4 \\
$2 \mathrm{~mm}<\mathrm{x}<20 \mathrm{~mm}$ & 24 & 19 \\
$20 \mathrm{~mm}<\mathrm{x} 40 \mathrm{~mm}$ & 43 & 55 \\
$\geq 40 \mathrm{~mm}$ & 26 & 22 \\
\hline
\end{tabular}

\subsection{Selección, muestreo y caracterización de suelo para bioensayo}

El suelo fue uno de los factores a tener en cuenta para evaluar la influencia del biochar sobre el cultivo, por lo que se seleccionaron suelos de dos parcelas distintas, ambas de manejo ecológico y con dos texturas diferentes. Se recogió una muestra compuesta de los primeros 30 $\mathrm{cm}$ en cada una de las parcelas y se secaron al aire en laboratorio. Se tomó una muestra 


\section{CONGRESO IBÉRICO DE AGROINGENIERÍA \\ X CONGRESSO IBÉRICO DE AGROENGENHARIA \\ 3 - 6 septiembre 2019, Huesca - España}

representativa de cada uno de ellos, y se realizó un análisis de fertilidad en un laboratorio acreditado. Por otra parte, también se realizó un análisis que permitió caracterizarlo biológicamente previamente al desarrollo del ensayo en invernadero.

La textura del suelo 1 resultó franco-arenosa (72,04\% arena total, 17,68\% limo y 10,28\% arcilla) y la del suelo 2 franco-arcillosa (33,11\% arena, 31,64\% limo, 35,25\% arcilla), lo que permitió establecer una diferencia importante en su comportamiento físico.

Se realizó una puesta a punto de la metodología de cultivo a través del seguimiento del crecimiento de plantas de cebada en ambos tipos de suelo tamizados a un tamaño inferior a 2 $\mathrm{mm}$ y mezclados con diferentes cantidades de gravilla fina esterilizada como sustrato inerte que permitiera evitar la compactación de los contenedores durante el ensayo. Para ambos suelos se optó por una mezcla $60 \%$ suelo $+40 \%$ gravilla fina que conformó el sustrato para el desarrollo del ensayo.

Los resultados del análisis de fertilidad físico-química de cada tipo de sustrato (suelo + gravilla) facilitados por el laboratorio externo se detallan en la Tabla 5.

Tabla 5. Resultados análisis de fertilidad de los sustratos utilizados en el ensayo (sustrato 1: suelo franco-arenoso + gravilla; sustrato 2: suelo franco-arcilloso 2 + gravilla)

\begin{tabular}{ccccc}
\hline Determinación realizada & Método & Unidad & Sustrato 1 & Sustrato 2 \\
\hline pH al agua $(1: 2,5)$ & MT-SUE-007 & & 8,0 & 8,2 \\
Conductividad eléctrica $(1: 5)$ & Electrometría & $\mathrm{dS} / \mathrm{m}$ & 0,4 & 0,2 \\
Materia orgánica oxidable & MT-SUE-002 & $\mathrm{g} / 100 \mathrm{~g}$ & 2,81 & 1,74 \\
Fósforo soluble (Olsen) & MT-SUE-003 & $\mathrm{mg} / \mathrm{kg}$ & 29 & 17 \\
Potasio soluble & MT-SUE-008 & $\mathrm{mg} / \mathrm{kg}$ & 254 & 130 \\
Magnesio & MT-SUE-008 & $\mathrm{mg} / \mathrm{kg}$ & 124 & 194 \\
Fósforo total (P2O5) & GRAVIMETRÍA & $\% \mathrm{p} / \mathrm{p}$ & $<0,38$ & $<0,38$ \\
Humedad retenida CC & GRAVIMETRÍA & $\% \mathrm{p} / \mathrm{p}$ & 9,94 & 17,05 \\
Humedad PMP & GRAVIMETRÍA & $\% \mathrm{p} / \mathrm{p}$ & 4,53 & 6,83 \\
\hline
\end{tabular}

Por otra parte el suelo se caracterizó biológicamente a traves del análisis cuantitativo de algunas poblaciones microbianas en el Centro de Investigaciones Agrarias y Tecnología Agroalimentaria de Aragón.

Para este análisis se siguieron las indicaciones dictadas por las normas que se detallan en la Tabla 6.

Tabla 6. Grupos microbianos analizados en este estudio, normativa y metodología

\begin{tabular}{|c|c|c|}
\hline Grupo microbiano & Metodología & Descripción \\
\hline $\begin{array}{c}\text { Preparación de } \\
\text { muestras }\end{array}$ & ISO 6887-1:1999 & $\begin{array}{l}\text { Preparación de muestras, suspensiones iniciales y diluciones } \\
\text { decimales para análisis microbiológico - Parte 1: Normas generales } \\
\text { para la preparación de suspensiones iniciales y diluciones decimales. }\end{array}$ \\
\hline $\begin{array}{l}\text { Microorganismos } \\
\text { aerobios mesófilos } \\
\text { totales }\end{array}$ & ISO 4833:2013 & $\begin{array}{l}\text { Método de enumeración de microorganismos - Parte 2: Colonias a } 30 \\
{ }^{\circ} \mathrm{C} \text { mediante técnica de extensión en superficie. }\end{array}$ \\
\hline Pseudomonas spp. & ISO 13720:21 & Enumeración de presuntas Pseudomonas spp. \\
\hline $\begin{array}{c}\text { Medio almidón } \\
\text { caseína [17] }\end{array}$ & $\begin{array}{r}\text { Almidón, } \\
\text { MgSO} 4 \cdot 7 \mathrm{H} 2 \\
\text { destilada, }\end{array}$ & $\begin{array}{l}\text { 10,0 g; Caseína, 0,3 g; KNO3, 2,0 g; NaCl, 2,0 g; K2HPO4, 2,0 g; } \\
\text { 2O, 0,05 g; CaCO3, 0,02 g; FeSO4·7H2O, 0,01 g; Agar, 15,0 g; Agua } \\
\text { 1,0 l. Ajustar pH a 7,2. Técnica de recuento de colonias a } 30{ }^{\circ} \mathrm{C} \text {. }\end{array}$ \\
\hline Mohos y Levaduras & $\begin{array}{l}\text { ISO } 21527- \\
1: 2008\end{array}$ & $\begin{array}{c}\text { Método para enumerar levaduras y mohos - Parte 1: técnica de } \\
\text { recuento de colonias en productos con una actividad de agua mayor a } \\
0,95 .\end{array}$ \\
\hline
\end{tabular}




\section{CONGRESO IBÉRICO DE AGROINGENIERÍA \\ X CONGRESSO IBÉRICO DE AGROENGENHARIA \\ 3 - 6 septiembre 2019, Huesca - España}

Tomando una muestra representativa de cada tipo de suelo, cada unidad analítica ( $25 \mathrm{~g})$ se colocó en una bolsa stomacher provista de filtro de celulosa. Para los grupos microbianos estudiados se utilizó, como diluyente de la unidad analítica, agua de peptona al 0,1\% (Merck, Darmstadt, Alemania) y se homogeneizó en un Stomacher Lab-Blender Circulator 400 (Seward Laboratory, Londres, Inglaterra) durante dos minutos a 260 r.p.m.

Estos medios de cultivo se prepararon siguiendo las indicaciones de etiquetado de los medios y de la normativa ISO 11133-1:200913.

Las medias de los valores obtenidos para cada grupo microbiano y la desviación típica, con tres repeticiones de cada muestra, y realizando conteos a los 4 y 7 días desde su incubación se muestran en la Tabla 7.

Tabla 7. Valores medios y desviación estándar del LogUFC/g para diferentes grupos microbianos en suelos de texturas diferentes (S1: suelo de textura franco-arenosa; S2: suelo de textura franco-arcillosa)

\begin{tabular}{cccc}
\hline Grupo microbiano & Unidad & Sustrato 1 & Sustrato 2 \\
\hline Aeróbios mesófilos totales & LogUFC/g & $6,59 \pm 0,90$ & $7,08 \pm 0,67$ \\
Mohos y levaduras & LogUFC/g & $4,75 \pm 0,65$ & $4,71 \pm 0,43$ \\
F F $^{\mathrm{a}}$ Enterobacteriaceae & LogUFC/g & $1,30 \pm 0,30$ & $1,90 \pm 0,20$ \\
G Pseudomonas $_{\text {Actinomicetos }}^{\text {LogUFC/g }}$ & $5,74 \pm 0,13$ & $5,96 \pm 0,34$ \\
LogUFC/g & $6,08 \pm 0,45$ & $6,45 \pm 0,27$ \\
\hline
\end{tabular}

Inicialmentea la carga microbiana total cultivable de los sustratos 1 y 2, es de 6,6 y 7,1 log $\mathrm{UFC} / \mathrm{g}$, respectivamente. Los recuentos más elevados en la rizosfera corresponden al $\mathrm{G}^{\mathrm{o}}$ Pseudomonas y Actinomicetos con alrededor de 6 u.log, seguido de la micobiota con 4,1 log $\mathrm{UFC} / \mathrm{g}$.

Además de las comunidades microbianas descritas anteriormente, se preparó un medio selectivo para hongos del género Trichoderma, TSM (Trichoderma Selective Medium) [18], como otro indicador de la fertilidad biológica de los suelos, resultando positiva su presencia en ambos suelos.

\subsection{Diseño experimental}

En el mes de noviembre de 2017 se instaló el bioensayo en invernadero. El cultivo seleccionado fue sorgo (Sorghum bicolor L.) y se estableció un diseño tri-factorial en bloques al azar que constó de los siguientes factores como variables independientes:

Factor 1: tipo de sustrato (suelo franco-arenoso + gravilla fina S1 y suelo franco-arcilloso + gravilla fina S2)

Factor 2: temperatura final de pirólisis del biochar utilizado $\left(400{ }^{\circ} \mathrm{C}-\mathbf{B 1}\right.$ y $\left.600{ }^{\circ} \mathrm{C}-\mathbf{B} 2\right)$

Factor 3: dosis de biochar aplicado (0 t/ha - D0 Control, 20 t/ha - D1 y 40 t/ha - D2).

El número de réplicas para cada combinación de factores fue de 5, por lo que se sembraron un total de 50 contenedores.

\subsection{Establecimiento y desarrollo del bioensayo}

Para el establecimiento del bioensayo se utilizaron bandejas de polietileno con doce alveolos de $650 \mathrm{~cm}^{3}$ de capacidad, $18 \mathrm{~cm}$ de profundidad y $64 \mathrm{~cm}^{2}$ de superficie superior cada uno.

Los alveolos se rellenaron con la correspondiente mezcla de sustrato (suelo + gravilla) a la que se añadió de forma homogénea la cantidad correspondiente de biochar.

El cultivo seleccionado para el desarrollo del ensayo fue sorgo (Sorghum bicolor L.), se pregerminaron semillas no tratadas químicamente, previamente desinfectadas en solución de 


\section{CONGRESO IBÉRICO DE AGROINGENIERÍA \\ X CONGRESSO IBÉRICO DE AGROENGENHARIA \\ 3 - 6 septiembre 2019, Huesca - España}

lejía en agua caliente al 1\% durante media hora. Tres semillas pregerminadas se coloraron en cada alveolo y se regaron de forma cuidadosa durante dos meses hasta que se realizó un aclareo que permitió mantener 1 planta/ alveolo.

El control de temperatura y humedad en el invernadero se realizó a través de una sonda de temperatura/humedad ambiental (HOBO Pro v2), que permitió ajustar las dosis y posturas de riego durante el ciclo del cultivo. Ambos tipos de suelo se regaron a capacidad de campo con distintas frecuencias de riego en función de la temperatura y la humedad registradas en el invernadero.

Durante los cuatro primeros meses de desarrollo las plantas fueron regadas únicamente con agua. En el cuarto mes se empezaron a notar síntomas de deficiencia de nutrientes por lo que se comenzó a regar con solución nutritiva de Hewitt [19].

El ensayo tuvo una duración de 13 meses en los que se completaron dos ciclos productivos completos, ya que una vez completado el primer ciclo (210 días desde siembra - D210), las plantas de sorgo se cortaron a unos $2 \mathrm{~cm}$ de la superifice del sustrato y rebrotaron un total de 42 de las 50 plantas inicicales (se mantuvo un número mínimo de 3 réplicas para cada uno de los tratamientos), completando un nuevo ciclo de cultivo (390 días desde siembra $-\mathrm{D}_{390}$ ).

\subsection{Medidas realizadas}

Tras completar ambos ciclos productivos, las plantas se cortaron y se tomaron las siguientes medidas:

- Peso fresco/ peso seco de la biomasa total (mediante secado en estufa hasta estabilización de peso a $70^{\circ} \mathrm{C}$ ). Se diferenció grano, parte aérea y sistema radicular; el dato referente al sistema radicular sólo en la segunda cosecha cuando se levantó por completo el ensayo.

- Tamaño de las plantas (altura desde base del tallo hasta inserción hoja bandera).

- Longitud hoja bandera (cuando la hubo).

Además de los parámetros anteriores, una vez levantado el ensayo se realizaron las siguientes medidas:

Parámetros biológicos:

- Análisis cuantitativo de poblaciones microbianas (metodología descrita en apartado 2.3).

\subsection{Análisis estadístico}

Los datos obtenidos fueron analizados estadísticamente mediante análisis multivariante y univariante combinado con el test de Tukey con un nivel de significación de 0,05. Se utilizó el programa IBM SPSS Statictics Versión 22.0.0.0.

\section{Resultados y discusión}

En este apartado se han sintetizado los principales resultados obtenidos de las medidas realizadas tras completar el segundo ciclo productivo del cultivo. Después de completar el primer ciclo (D210), no se observaron diferencias significativas en los parámetros morfológicos y productivos analizados según el tratamiento (dosis y tipo de biochar) aplicado.

En este sentido, cabe destacar que en la mayoría de la bibliografía consultada (se citan sólo algunos ejemplos en este texto) $[20,21,22]$ los efectos del biochar sobre el desarrollo del cultivo de sorgo o especies de crecimiento similar como el maíz, y sobre las propiedades del suelo se han visto reflejados en ensayos de mucha menor duración. Además, en el caso concreto de este estudio, por tratarse de un cultivo con capacidad de rebrote, las raíces del mismo han estado un tiempo más largo en contacto con la enmienda en el suelo, lo que resalta la necesidad de profundizar en las diferencias que pueden existir entre los espacios de tiempo a los que se somete a contacto con el biochar a determinados cultivos. 


\subsection{Parametros productivos}

Los valores medios y el intervalo de confianza de los datos relativos a peso seco de la biomasa total producida $\left(\mathrm{P}_{\mathrm{T}}\right)$, peso seco de la biomasa de la parte aérea $\left(\mathrm{P}_{\mathrm{A}}\right)$, peso seco de grano $\left(\mathrm{P}_{\mathrm{G}}\right)$ y peso seco de raíz $\left(\mathrm{P}_{\mathrm{R}}\right)$ se muestran en la Figura 1 ( $\mathrm{a}, \mathrm{b}$, c y d respectivamente). En las gráficas se observa la influencia del biochar y la dosis de aplicación combinando los efectos de ambas texturas de sustrato. Estos valores se han considerado interesantes entre las medidas realizadas al finalizar el ciclo del cultivo $\left(\mathrm{D}_{390}\right)$ ya que están relacionados directamente con el interés agronómico del cultivo, tanto desde el punto de vista de la producción de grano como de forraje o en el caso de la raíz, planteando el objetivo de producir una mayor cantidad de biomasa subterránea capaz de explorar un mayor volumen de suelo e incluso actuar en situaciones de sequía o compactación.

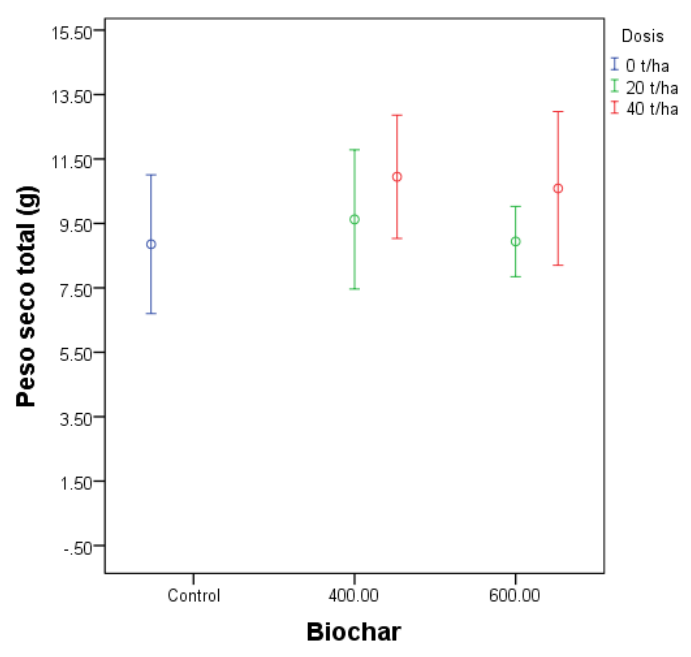

a.

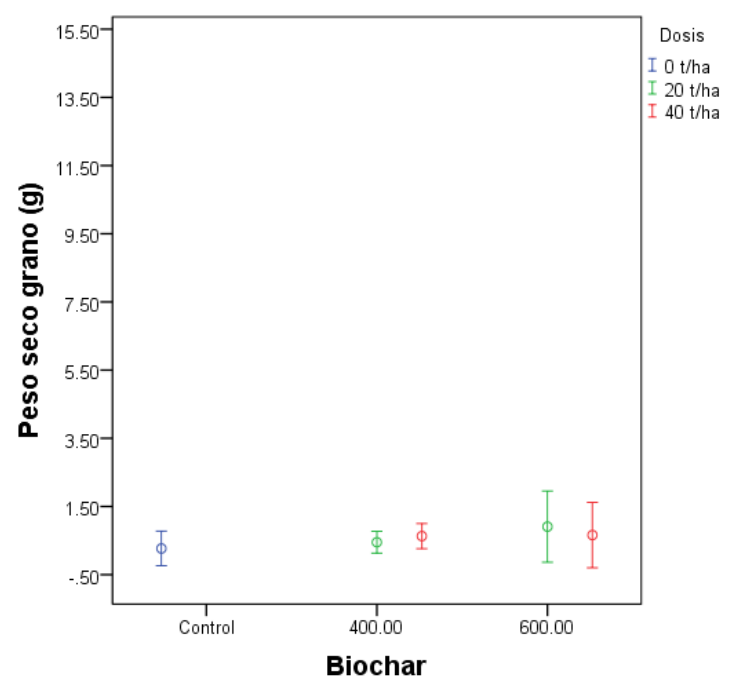

c.

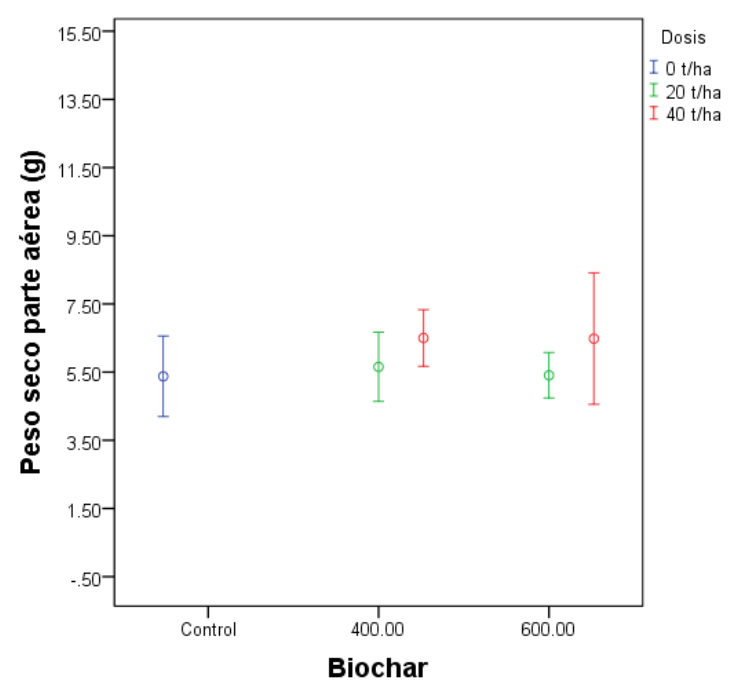

b.

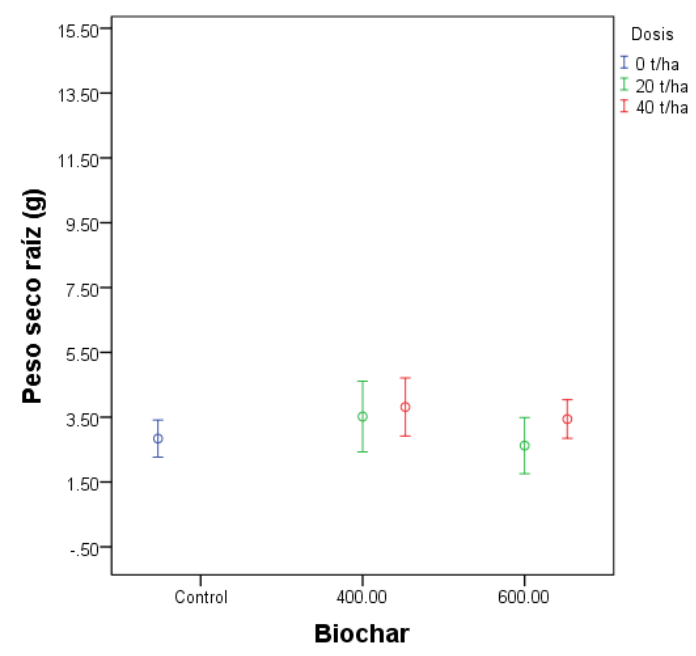

d.

Figura 1. Valores medios e intervalo de confianza al $95 \%$ de los pesos secos generados por el cultivo bajo diferentes dosis de biochar a $400{ }^{\circ} \mathrm{C}$ y a $600{ }^{\circ} \mathrm{C}$ (a: peso seco total de la biomasa, b: peso seco total de la parte aérea, c: peso seco total de grano, d: peso seco total de la raíz) 


\section{CONGRESO IBÉRICO DE AGROINGENIERÍA \\ X CONGRESSO IBÉRICO DE AGROENGENHARIA \\ 3 - 6 septiembre 2019, Huesca - España}

Los resultados del análisis multivariante de los datos anteriores se muestras en la Tabla 8. Se observa que el tipo de suelo es el factor fundamental que afecta significativamente a los valores obtenidos en peso seco total de las plantas, peso seco de la parte aérea y peso seco de la raíz.

Tabla 8. Resultados del análisis multivariante del efecto del tipo de suelo, tipo de biochar y dosis de aplicación sobre algunos parámetros productivos en un cultivo de sorgo bajo condiciones controladas. Nivel de significación $\mathrm{p} \leq 0,05$

(PT: peso seco total; PA: peso seco parte aérea; PG: peso seco grano; PR: peso seco raíz)

\begin{tabular}{|c|c|c|c|c|c|c|}
\hline Factor & $\begin{array}{c}\text { Variable } \\
\text { dependiente }\end{array}$ & $\begin{array}{l}\text { Tipo III de } \\
\text { suma de } \\
\text { cuadrados }\end{array}$ & gl & $\begin{array}{l}\text { Cuadrático } \\
\text { promedio }\end{array}$ & F & Sig. \\
\hline \multirow[t]{4}{*}{ Biochar } & PT & 7,220 & 1 & 7,220 & 2,095 & 0,158 \\
\hline & PA & 1,233 & 1 & 1,233 & 0,815 & 0,373 \\
\hline & PG & 0,140 & 1 & 0,140 & 0,179 & 0,675 \\
\hline & PR & 3,806 & 1 & 3,806 & 5,156 & 0,030 \\
\hline \multirow[t]{4}{*}{ Sustrato } & PT & 97,984 & 1 & 97,984 & 28,430 & 0,000 \\
\hline & PA & 27,973 & 1 & 27,973 & 18,499 & 0,000 \\
\hline & PG & 3,227 & 1 & 3,227 & 4,125 & 0,051 \\
\hline & PR & 4,194 & 1 & 4,194 & 5,682 & 0,023 \\
\hline \multirow[t]{4}{*}{ Dosis } & PT & 19,520 & 1 & 19,520 & 5,664 & 0,023 \\
\hline & PA & 6,471 & 1 & 6,471 & 4,279 & 0,047 \\
\hline & PG & 0,023 & 1 & 0,023 & 0,029 & 0,865 \\
\hline & PR & 4,104 & 1 & 4,104 & 5,561 & 0,025 \\
\hline \multirow[t]{4}{*}{ Biochar*Sustrato } & PT & 8,953 & 1 & 8,953 & 2,598 & 0,117 \\
\hline & PA & 0,063 & 1 & 0,063 & 0,042 & 0,839 \\
\hline & PG & 0,142 & 1 & 0,142 & 0,182 & 0,673 \\
\hline & PR & 8,218 & 1 & 8,218 & 11,134 & 0,002 \\
\hline \multirow[t]{4}{*}{ Biochar*Dosis } & PT & 0,411 & 1 & 0,411 & 0,119 & 0,732 \\
\hline & PA & 0,100 & 1 & 0,100 & 0,066 & 0,799 \\
\hline & PG & 0,903 & 1 & 0,903 & 1,155 & 0,291 \\
\hline & PR & 0,391 & 1 & 0,391 & 0,529 & 0,472 \\
\hline \multirow[t]{4}{*}{ Sustrato*Dosis } & PT & 1,573 & 1 & 1,573 & 0,456 & 0,504 \\
\hline & PA & 1,579 & 1 & 1,579 & 1,044 & 0,314 \\
\hline & PG & 1,133 & 1 & 1,133 & 1,449 & 0,238 \\
\hline & PR & 1,139 & 1 & 1,139 & 1,543 & 0,223 \\
\hline \multirow[t]{4}{*}{ Biochar*Sustrato*Dosis } & PT & 2,523 & 1 & 2,523 & 0,732 & 0,399 \\
\hline & PA & 2,136 & 1 & 2,136 & 1,413 & 0,243 \\
\hline & PG & 0,509 & 1 & 0,509 & 0,651 & 0,426 \\
\hline & PR & 0,344 & 1 & 0,344 & 0,466 & 0,500 \\
\hline
\end{tabular}

A su vez se observan diferencias significativas derivadas del efecto de la aplicación de biochar sobre el peso de la raíz y de la dosis sobre el peso total de las plantas, el peso de la parte aérea y el peso de la raíz. En la Figura 2 ( $a$ y b) se observa el distinto comportamiento del desarrollo de la raíz en función del tipo de suelo, biochar y dosis siendo mucho más acusadas las diferencias en el sustrato franco-arenoso que en el franco-arcilloso. 


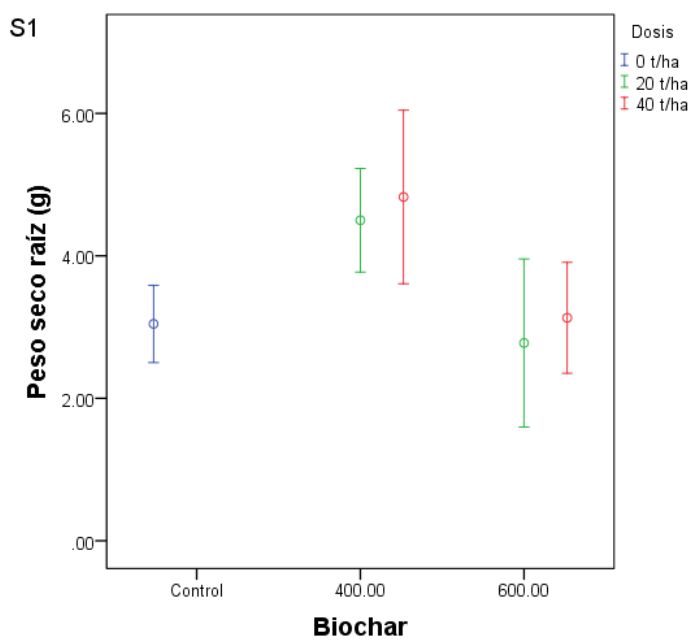

a.

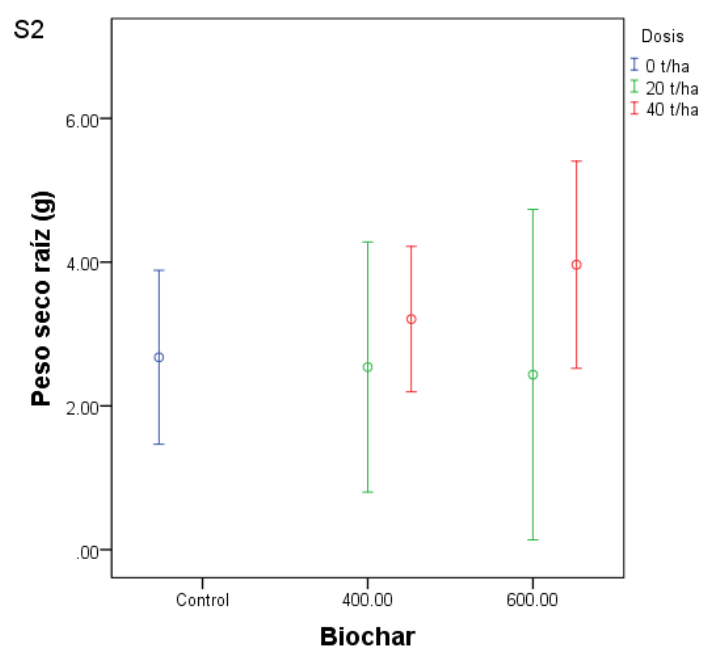

b.

Figura 2. Valores medios e intervalo de confianza al 95\% del peso seco total de la raíz generada por el cultivo diferentes dosis de biochar a $400{ }^{\circ} \mathrm{C}$ y a $600{ }^{\circ} \mathrm{C}$ (a: peso seco total de la raíz en el sustrato 1 (S1) de origen franco-arenoso, b: peso seco total de la raíz en el sustrato 2 (S2) de origen franco-arcilloso)

El análisis de datos univariante para cada tipo de suelo muestra diferencias significativas en el peso seco de la raíz para el caso del sustrato 1 de origen franco-arenoso con adición de biochar producido a $400^{\circ} \mathrm{C}$, el peso seco de las raíces en este tratamiento se incrementó en un $47,5 \%$ respecto al tratamiento control. El análisis no muestra diferencias significativas $(p=0,817)$ en el caso del sustrato 2 de origen franco-arcilloso. Los resultados del análisis combinado con el test de Tukey para un nivel de significación $\mathrm{p} \leq 0,05$ se detallan en la Tabla 9.

Tabla 9. Resultados del análisis univariante del efecto del tipo de biochar sobre el peso seco de la raíz en un cultivo de sorgo bajo condiciones controladas bajo dos sustratos de cultivo diferentes. Nivel de significación $\mathrm{p} \leq 0,05$.

(S1: suelo franco-arenoso + gravilla; S2: suelo franco-arcilloso + gravilla; B400: biochar $400{ }^{\circ} \mathrm{C}$; B600: biochar $600^{\circ} \mathrm{C}$ )

\begin{tabular}{|c|c|c|c|c|c|c|c|}
\hline \multirow{2}{*}{ HSD Tukey } & \multirow{2}{*}{ Biochar } & \multirow{2}{*}{ Biochar } & \multirow{2}{*}{$\begin{array}{c}\text { Diferencia de } \\
\text { medias }\end{array}$} & \multirow{2}{*}{$\begin{array}{c}\text { Error } \\
\text { estándar }\end{array}$} & \multirow{2}{*}{ Sig. } & \multicolumn{2}{|c|}{ I.C. $95 \%$} \\
\hline & & & & & & L.I & L.S \\
\hline \multirow[t]{6}{*}{ S1 } & Control & B400 & $-1,595$ & 0,39492 & 0,002 & $-2,6029$ & $-0,5871$ \\
\hline & & B600 & 0,0920 & 0,37275 & 0,967 & $-0,8593$ & 1,0433 \\
\hline & B400 & Control & 1,5950 & 0,39492 & 0,002 & 0,5871 & 2,6029 \\
\hline & & B600 & 1,6870 & 0,31050 & 0,000 & 0,8946 & 2,4794 \\
\hline & B600 & Control & $-0,0920$ & 0,37275 & 0,967 & $-1,0433$ & 0,8593 \\
\hline & & B400 & $-1,6870$ & 0,31050 & 0,000 & $-2,4794$ & $-0,8946$ \\
\hline \multirow[t]{6}{*}{ S2 } & Control & B400 & $-0,2351$ & 0,61616 & 0,923 & $-1,8077$ & 1,3374 \\
\hline & & B600 & $-0,4140$ & 0,64684 & 0,800 & $-2,0648$ & 1,2368 \\
\hline & B400 & Control & 0,2351 & 0,61616 & 0,923 & $-1,3374$ & 1,8077 \\
\hline & & B600 & $-0,1789$ & 0,55671 & 0,945 & $-1,5997$ & 1,2419 \\
\hline & B600 & Control & 0,4140 & 0,64684 & 0,800 & $-1,2368$ & 2,0648 \\
\hline & & B400 & 0,1789 & 0,55671 & 0,945 & $-1,2419$ & 1,5997 \\
\hline
\end{tabular}




\section{CONGRESO IBÉRICO DE AGROINGENIERÍA \\ X CONGRESSO IBÉRICO DE AGROENGENHARIA \\ 3 - 6 septiembre 2019, Huesca - España}

La proliferación del volumen radicular observada en el sustrato franco-arenoso con la adición de biochar producido a $400^{\circ} \mathrm{C}$ coincide con los resultados observados en otros estudios $[23,24]$ con biochar de distintos orígenes. Además, la tendencia creciente en el resto de parámetros productivos con la adición en dosis crecientes de biochar sugiere que el biochar producido a $400{ }^{\circ} \mathrm{C}$ podría tener efectos positivos mayores que el biochar producido a $600{ }^{\circ} \mathrm{C}$ en el desarrollo de las plantas en suelos franco-arenosos. Sin embargo, la ausencia de diferencias significativas para los parámetros estudiados en ambos tipos de sustrato (relacionadas con el vigor y la producción de biomasa total de las plantas) plantea la necesidad de estudiar en mayor profundidad las dosis de este tipo de biochar aplicadas al suelo, así como la necesidad de analizar a nivel práctico la dificultad de aplicar en un suelo dosis mayores a las planteadas (20 - $40 \mathrm{t} / \mathrm{ha}$ ), ya que debido a la baja densidad aparente del producto podrían resultar poco razonables. Otro componente a tener en cuenta es la granulometría del biochar, ya que en este estudio se ha optado por una distribución de tamaños derivada de la aproximación a un proceso de aplicación real en parcela; con una distribución diferente de tamaños los resultados podrían variar y en ese sentido habría que profundizar en mayor medida en el efecto del procesado del biochar previo a su aplicación al suelo.

\subsection{Poblaciones microbianas}

Respecto al análisis cuantitativo de poblaciones microbianas, la Figura 3 muestra los valores medios de unidades formadoras de colonias y la desviación estándar de los grupos microbianos analizados para cada uno de los tratamientos.

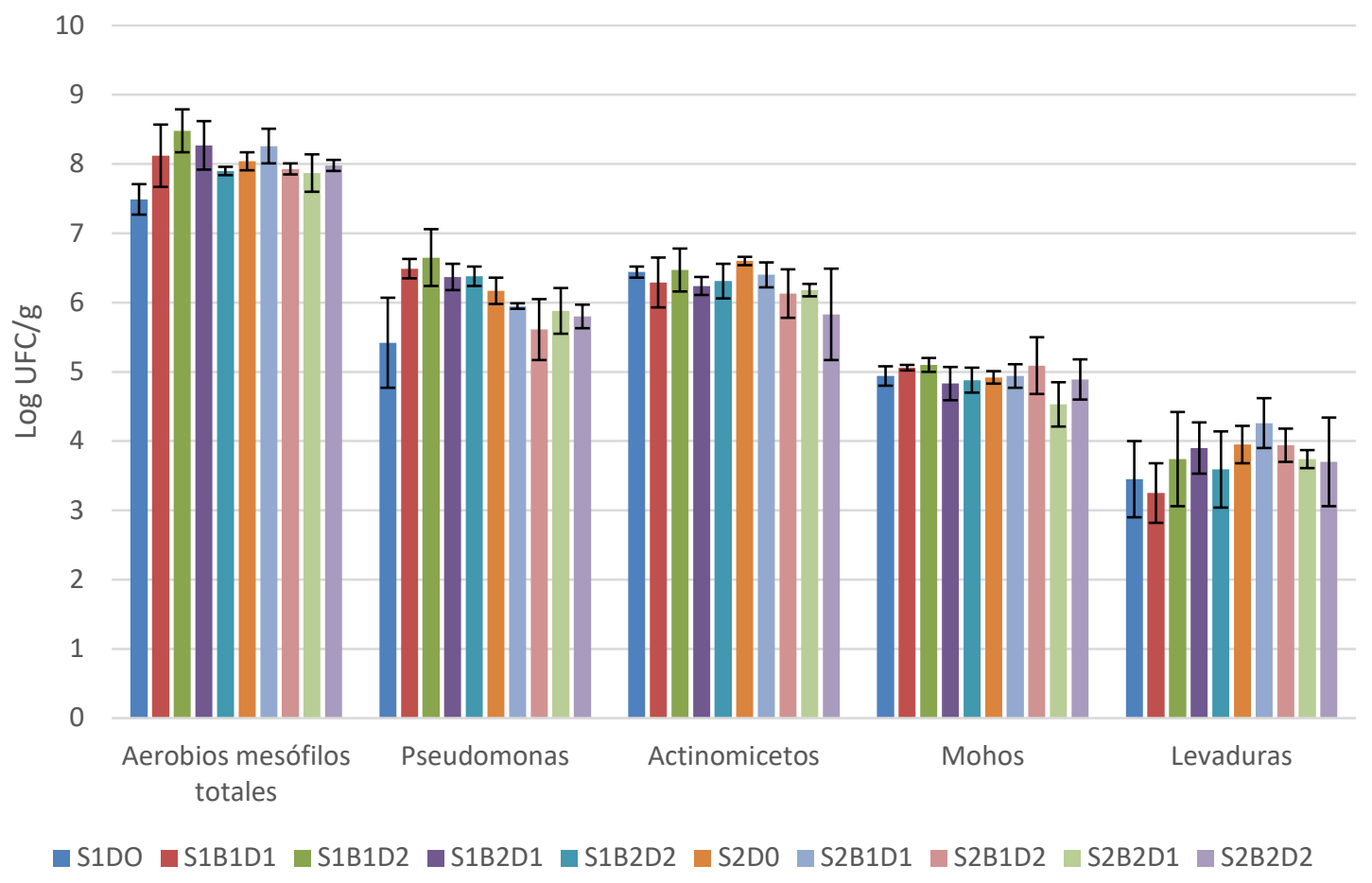

Figura 3. Valores medios de log. UFC y desviación estándar de diferentes grupos microbianos para cultivo de sorgo bajo diferentes dosis de biochar producido a dos temperaturas y dos tipos de sustrato (S1: sustrato 1 franco-arenoso; S2: sustrato 2 franco-arcilloso; B1: biochar $400{ }^{\circ} \mathbf{C}$; B2: biochar $600^{\circ} \mathrm{C}$; D1: dosis $10 \mathrm{t} / \mathrm{ha}$; D2: dosis $40 \mathrm{t} / \mathrm{ha}$ ) 


\section{CONGRESO IBÉRICO DE AGROINGENIERÍA \\ X CONGRESSO IBÉRICO DE AGROENGENHARIA \\ 3 - 6 septiembre 2019, Huesca - España}

Los recuentos telúricos de todos los grupos microbianos se han mantenido estables a lo largo del periodo de ensayo. Aunque la tendencia, ha sido a aumentar ligeramente, los recuentos obtenidos han sido inferiores a $1 \mathrm{u} . \log$ respecto al estado inicial. Si que es cierto, que especies microbianas específicas si que podrían ser mayoritarias debido a una mejor adaptación a las condiciones, suelo y sistema radicular, hecho que no se ha comprobado en este trabajo.

Los resultados obtenidos en los recuentos de estos 2 sustratos han resultado similares a los de otros autores, en el caso de los microorganismos aerobios mesófilos totales, 7,6 u.log/g [25]; y para la micobiota, 4,6 u.log/g [25] y 3,9 u.log/g [26].

Respecto a las diferencias entre tratamientos, la Tabla 10 muestra los resultados del análisis multivariante del efecto del tipo de sustrato, tipo de biochar y dosis de aplicación sobre el tamaño de algunas poblaciones microbianas.

Tabla 10. Resultados del análisis multivariante del efecto del tipo de suelo, tipo de biochar y dosis de aplicación sobre el tamaño de algunas poblaciones microbianas. Se muestran los factores en los que se observaron diferencias significativas. Nivel de significación $\mathrm{p} \leq 0,05$

(AMT: aerobios mesófilos totales; PS: Pseudomonas sp.; ACT: actinomicetos; M: mohos; L: levaduras)

\begin{tabular}{ccccccc}
\hline Factor & $\begin{array}{c}\text { Variable } \\
\text { dependiente }\end{array}$ & $\begin{array}{c}\text { Tipo III de } \\
\text { suma de } \\
\text { cuadrados }\end{array}$ & gl & $\begin{array}{c}\text { Cuadrático } \\
\text { promedio }\end{array}$ & F & Sig. \\
\hline Biochar & AMT & 0,220 & 1 & 0,220 & 3,460 & 0,078 \\
& PS & 0,027 & 1 & 0,027 & 0,264 & 0,613 \\
& ACT & 0,204 & 1 & 0,204 & 2,211 & 0,153 \\
& M & 0,419 & 1 & 0,419 & 8,054 & 0,010 \\
& L & 0,023 & 1 & 0,023 & 0,113 & 0,740 \\
\hline Biochar*Sustrato*Dosis & AMT & 0,505 & 1 & 0,505 & 7,922 & 0,011 \\
& PS & 0,063 & 1 & 0,063 & 0,608 & 0,445 \\
& ACT & 0,001 & 1 & 0,001 & 0,005 & 0,942 \\
& M & 0,016 & 1 & 0,016 & 0,298 & 0,591 \\
& L & 0,429 & 1 & 0,429 & 2,078 & 0,165 \\
\hline
\end{tabular}

Se observan diferencias en el grupo de aeróbios mesófilos totales afectados significativamente $(\mathrm{p}=0,011)$ por la combinación de los tres factores independientes, además el test de Tukey para este grupo detectó un incremento significativo $(\mathrm{p}=0,007)$ por la adición de biochar, independientemente de la temperatura de producción y de la dosis de aplicación. El análisis univariante diferenciando ambos tipos de suelo, combinado con el test de Tukey, mostró que estas diferencias fueron significativas en ambos tipos de suelo.

Los hongos filamentosos o mohos se observan afectados significativamente $(\mathrm{p}=0,010)$ por el tipo de biochar. El test de Tukey reflejó únicamente un incremento significativo $(p=0.026)$ con el biochar producido a $600{ }^{\circ} \mathrm{C}$, independientemente de la dosis. El análisis univariante diferenciando por tipo de suelo permitió establecer que estas diferencias sólo se produjeron de forma significativa $(p=0,031)$ en el suelo franco-arenoso.

En el resto de grupos microbianos no se han podido determinar estas diferencias. Un estudio a más largo plazo, o en cultivos plurianuales podría determinar mejor como es la evolución de los diferentes grupos microbianos con estas mismas condiciones.

Además de la cuantificación de microorganismos expuesta en este estudio como indicador biológico se han realizado medidas de otros indicadores biológicos que permitirán complementar estos datos. 


\section{CONGRESO IBÉRICO DE AGROINGENIERÍA \\ X CONGRESSO IBÉRICO DE AGROENGENHARIA \\ 3 - 6 septiembre 2019, Huesca - España}

\section{Conclusiones}

En base a todo lo expuesto en el presente trabajo se plantean las siguientes conclusiones:

El sarmiento de viña se plantea como un recurso de interés para la producción de biochar, por la facilidad de transporte y procesado, la aptitud de su estructura en relación al proceso de pirólisis así como por sus características finales.

Los efectos del biochar, para las condiciones del ensayo planteado, han sido muy diferentes en función del tipo de suelo analizado, habiéndose constatado un mayor efecto sobre el desarrollo radicular en el tipo de sustrato que tenía como componente un suelo franco-arenoso. El tipo de biochar y dosis que han tenido unos efectos mayores sobre el desarrollo del cultivo ha sido el biochar producido a $400{ }^{\circ} \mathrm{C}$, independientemente de la dosis aplicada. En base a lo anterior, se hace necesario ajustar en mayor medida las dosis de aplicación del biochar y la granulometría del mismo en el momento de su aplicación al suelo, ya que se ha observado una tendencia al incremento del desarrollo del cultivo relacionada con el incremento de la dosis para ambos tipos de suelo.

El tamaño de las poblaciones microbianas de microorganismos mesófilos totales y hongos filamentosos se ha visto incrementado con la adición de biochar. Sin embargo, la elección del análisis cuantitativo de poblaciones microbianas como indicador biológico en este estudio debe ser complementada con un mayor número de indicadores biológicos que puedan resultar más sensibles a la adición de biochar.

\section{Referencias}

1. García, C.; Rosas, J.G.; Sánchez, M.E., Pascual, J.A., Hernández, M.T. Cap. 8: Enmiendas orgánicas y de nueva generación: biochar y otras biomoléculas. En De residuo a recurso: el camino hacia la sostenibilidad. III. Recursos orgánicos. Aspectos agronómicos y medioambientales. Red española de compostaje. 2014, 182 pp.

2. Whitman, T., Lehmann, J. Biochar-One way forward for soil carbon in offset mechanisms in Africa? Environ. Sci. Policy 2009, 12, $1024-1027$.

3. Lehmann, J., Gaunt, J., Rondon, M. Bio-char sequestration in terrestrial ecosystems: a review. Mit. Adapt. Strateg. Glob. Chang. 2006, 11: 403-427.

4. Fowles, M. 2007. Black carbon sequestration as an alternative to bioenergy. Biomass Bioenergy 2007, $31(6), 426-432$.

5. Gaunt, J.L., Lehmann, J. Energy balance and emissions associated with biochar sequestration and pyrolysis bioenergy production. Environ. Sci. Technol. 2008, 42 (11), $4152-4158$.

6. Méndez, A., Terradillos, M., Gascó, G. Physicochemical and agronomic properties of biochar from sewage sludge pyrolysed at different temperatures. J. Anal. Appl. Pyrolysis 2013, 102, $124-130$.

7. Enders, A., Hanley, K., Whitman, T. Joseph, S., Lehman, J. Characterization of biochars to evaluate recalcitrance and agronomic performance. Bioresour. Technol. 2012, 114, $644-653$.

8. Manyà, J.J., Ortigosa, M.A., Laguarta, S., Manso, J.A. Experimental study on the effect of pyrolysis pressure, peak temperature, and particle size on the potential stability of vine shoots-derived biochar. Fuel 2014, 133, 163 - 172.

9. Antal, M.J., Allen, S.G., Dai, X., Shimizu, B., Tam, M.S., Gronli, M. Attainment of the theoretical yield of carbon from biomass. Ind. Eng. Chem. Res. 2000, 39, $4024-4031$.

10. Manyà, J.J., Azuara, M., Manso, J.A. Biochar production through slow pyrolysis of different biomass materials: Seeking the best operating conditions. Biomass bioenergy 2018, 117, 115 - 123.

11. Zhao, L., Cao, X., Masek, O. Zimmerman, A. Heterogeneity of biochar properties as a function of the feedstock sources and production temperatures. J. Hazard. Mater. 2013, 256 - 257: 1 - 9.

12. Peralbo-Molina, A. Luque de Castro, M.D. Potential of residues from the Mediterranean agriculture and agrifood industry. Trends Food Sci. Technol. 2013, 32, $16-24$.

13. Duca, D., Toscano, G., Pizzi, A., Rossini, G., Fabrizi, S. Lucesoli, G., Servili, A., Mancini, V., Romanazzi, G., Mengarelli, C. Evaluation of the characteristics of vineyard pruning residues for 


\section{CONGRESO IBÉRICO DE AGROINGENIERÍA \\ X CONGRESSO IBÉRICO DE AGROENGENHARIA \\ 3- 6 septiembre 2019, Huesca - España}

energy applications: effect of different copper-based treatments. J. Agric. Eng. 2016, XLVII, 497, 22 27.

14. Demirbas, A. Effects of temperature and particle size on bio-char yield from pyrolysis of agricultural residues. J. Anal. Appl. Pyrol. 2014, 72, 243 - 248.

15. Shen, J. Wang, X., García-Pérez, M., Mourant, D., Rhodes, M.J., Li, C. Effects of particle size on the fast pyrolysis of oil mallee woody biomass. Fuel 2009, 88, $1810-1817$.

16. Greco, G., Videgain, M., Di Stasi, C., González, B., Manyà, J.J. Evolution of the mass-loss rate during atmospheric and pressurized slow pyrolysis of wheat straw in a bench-scale reactor. J. Anal. Appl. Pyrol. 2018. 136, 18-26.

17. Kuster, E., Williams, S.T. Selection of media for isolation of Streptomycetes. Nature 1964, 202, 928 929.

18. Askew, D.J., Laing, M.D. An adapted selective medium for the cuantitative isolation of Trichoderma species. J. Plant Pathol. 1993, 42, 686 - 690.

19. Hewitt, E.J. Sand and water culture methods used in the study of plant nutrition. Technical communication. Commonwealth Agricultural Bureaux, Farnham Royal. 1969, 22 (2 $2^{\mathrm{a}}$ edición revisada), $547 \mathrm{pp}$.

20. Brennan, A., Moreno, E., Alburquerque, J.A., Knapp, C.W., Switzer, C. Effects of biochar and activated carbon amendment on maize growth and the uptake and measured availability of polycyclic aromatic hydrocarbons (PAHs) and potentially toxic elements (PTEs). Environ. Pollut. 2014, 193, $79-87$.

21. Hairani, A., Osaki, M., Watanabe, T. Effect of biochar application on mineral and microbial properties of soils growing different plant species. J. Soil Sci. Plant Nutr. 2016, 62, 519-525.

22. Rafique, M., Sultan, T., Ortas, I., Chaudhary, H.J. Enhancement of maize plant growth with inoculation of phospate_slubilizing bacteria and biochar amendment in soil. J. Soil Sci. Plant Nutr. 2017, 63, 460-469.

23. Olmo, M., Villar, R., Salazar, P., Alburquerque, J.A. Changes in soil nutrient availability explain biochar's impact on wheat root development. Plant Soil 2016, 399, 333 - 343.

24. Zhang, Y., Chen, H., Ji, G., Zhang, Y., Xiang, J., Anwar, S., Zhu, D. Effect of rice-straw application on rice (Oryza sativa L.) root growth and nitrogen utilization in acidified paddy soil. Int. J. Agric. Biol. 2018, 20, 11, 2529 - 2536.

25. Arif, M.S., Riaz, M., Shahzad, S.M., Yasmeen, T., Ashraf, M., Siddique, M., Mubarik, M.S., Bragazza, L. \& Buttler, A. Fresh and composted industrial sludge restore soil functions in surface soil of degraded agricultural land. Sci. Total Environ. 2018, 619-620, 517-527.

26. Saravanakumara, K., Kathiresana, K., MubarakAlib, D., Kayalvizhia, K., Rajendranc, N., Hemalathab, S. \& Chend, J. Soil-microbial communities indexing from mangroves rhizosphere and barren sandy habitats. Physiol. Mol. Plant Pathol. 2018, 104, 58-68. 